\title{
Prevention and modulation of aminoglycoside ototoxicity (Review)
}

\author{
GIANPAOLO PERLETTI $^{1}$, ANNE VRAL ${ }^{2}$, MARIA CRISTINA PATROSSO $^{3}$, EMANUELA MARRAS ${ }^{1}$, \\ ISABELLA CERIANI $^{1}$, PETRA WILLEMS ${ }^{2}$, MAURO FASANO ${ }^{1}$ and VITTORIO MAGRI ${ }^{4}$
}

\begin{abstract}
${ }^{1}$ Department of Structural and Functional Biology, Laboratory of Toxicology and Pharmacology, and Center of Neuroscience (MF), Università degli Studi dell'Insubria, Busto Arsizio, Varese, Italy; ${ }^{2}$ Department of Anatomy, Embryology, Histology and Medical Physics, Ghent University, Ghent, Belgium; ${ }^{3}$ Medical Genetics Laboratory, Medicine Laboratory

Department, Niguarda Ca'Granda Hospital; ${ }^{4}$ Hospital Istituti Clinici di Perfezionamento, Milan, Italy
\end{abstract}

Received November 6, 2007; Accepted November 29, 2007

\begin{abstract}
More than 60 years after their isolation and characterization, aminoglycoside (AG) antibiotics remain powerful agents in the treatment of severe gram-negative, enterococcal or mycobacterial infections. However, the clinical use of AGs is hampered by nephrotoxicity and ototoxicity, which often develop as a consequence of prolonged courses of therapy, or of administration of increased doses of these drugs. The discovery of non-ototoxic antibacterial agents, showing a wider spectrum of activity, has gradually decreased the use of AGs as first line antibiotics for many systemic infections. However, AGs are now undergoing an unexpected revival, being increasingly indicated for the treatment of severe emerging infections caused by organisms showing resistance to most first-line agents (e.g., multidrug-resistant tuberculosis, complicated nosocomially-acquired acute urinary tract infections). Increasing adoption of aminoglycosides poses again to scientists and physicians the problem of toxicity directed to the kidneys and to the inner ear. In particular, aminoglycoside-induced deafness can be profound and irreversible, especially in genetically predisposed patients. For this reason, an impressive amount of molecular strategies have been developed in the last decade to counteract the ototoxic
\end{abstract}

Correspondence to: Dr Gianpaolo Perletti, Dipartimento di Biologia Strutturale e Funzionale, Università degli Studi dell'Insubria, Via A. Da Giussano 12, I-21052 Busto A., Italy

E-mail: gianpaolo.perletti@uninsubria.it

Abbreviations: AG, aminoglycoside; NAUTI, nosocomiallyacquired urinary tract infections; rRNA, ribosomal RNA; NSHL, non-syndromic hearing loss; mtDNA, mitochondrial DNA; ROS, reactive oxygen species; MDD, multiple daily dosing; SDD, single daily dosing

Key words: aminoglycoside, ototoxicity, non-syndromic hearing loss, streptomycin, paromomycin, gentamicin, neomycin, aspirin effect of aminoglycosides. The present article overviews: i) the molecular mechanisms by which aminoglycosides exert their bactericidal activity, ii) the mechanisms whereby AGs exert their ototoxic activity in genetically-predisposed patients, iii) the drugs and compounds that have so far proven to prevent or modulate AG ototoxicity at the preclinical and/or clinical level, and iv) the dosage regimens that have so far been suggested to decrease the incidence of episodes of AG-induced ototoxicity.

\section{Contents}

1. Aminoglycoside antibiotics: a revival?

2. Bactericidal action of aminoglycosides

3. Hearing loss induced by aminoglycosides

4. Mutations predisposing to non-syndromic hearing loss (NSHL) and aminoglycoside ototoxicity

5. Prevention and modulation of aminoglycoside-induced ototoxicity

6. Dose and regimen optimization

7. Conclusions

\section{Aminoglycoside antibiotics: a revival?}

Aminoglycoside (AG) antibiotics have been extensively used for the prophylaxis and the treatment of a wide variety of systemic infections, for the outstanding features displayed by these antibacterial agents, i.e., the concentration-dependent bactericidal activity, the post-antibiotic effect, the favorable pharmacokinetic profile, and the strong synergism with other antibiotics such as vancomycin and B-lactams (1). More than 60 years after their isolation and characterization, these drugs remain powerful tools for treatment of severe gram-negative, enterococcal or mycobacterial infections. AGs are usually administered in combination with $\beta$-lactam antibiotics for a variety of systemic infections (e.g., bacterial endocarditis or various pseudomonal infections), but are also used as monotherapy for infections of the urinary tract. Streptomycin, the first AG isolated and adopted for human therapy (Fig. 1), 
is still indicated for treatment of gentamicin-resistant enterococcal infections. Amikacin, one of the most widely adopted members of the AG family, is useful against gentamicinresistant gram-negative organisms and in the treatment of infections caused by nontuberculous mycobacteria.

The clinical use of aminoglycosides has always been hampered by nephrotoxicity and by the relatively high incidence of severe ototoxicity, which often develops as a consequence of prolonged courses of therapy, or after administration of increased doses of these agents. The discovery of a number of non-ototoxic agents (e.g., fluoroquinolones), characterized by a larger spectrum of antibacterial activity and by optimal bioavailability following oral administration, has gradually decreased the adoption of AGs as first-line antibiotics for the treatment of a number of infectious diseases. However, the emergence of severe, life-threatening, drug resistant infections has recently restored a new interest in AGs.

At the time of its discovery by Schatz and Waksman (1943) (2), streptomycin was used as the first available antibiotic effective against Mycobacterium tuberculosis. AGs were subsequently replaced by alternative antitubercular drugs such as isoniazid, discovered in 1952, and rifampicin (1963). In the last decade, highly-resistant, life-threatening diseases, like multidrug-resistant tuberculosis (MDR-TB), have compelled physicians and researchers to establish in a very short time alternative therapeutic protocols. MDR-TB occurs when the causative agent shows resistance to, at least, isoniazid and rifampicin, two fundamental first-line components of the standard therapeutic regimen for drug-susceptible M. tuberculosis. In recommended therapeutic regimens, AGs are always indicated for treatment of MDR-TB, in combination with at least two other second-line anti-tubercular agents. Suggested protocols for MDR-TB rank such agents in order of importance as follows: i) aminoglycosides/ capreomycin and levofloxacin; ii) ethionamide/prothionamide and ofloxacin/ciprofloxacin; iii) PAS; iv) cycloserine; v) Blactams; vi) clarithromycin, linezolid and clofazimine (3). Usually, long-term treatment (18-24 months) with a combination of ethambutol, pyrazinamide, a fluoroquinolone and amikacin is indicated in the presence of resistance to isoniazid and rifampicin, associated or not with resistance to streptomycin (3).

Besides their increased use as antitubercular agents, AGs are being indicated for the treatment of complicated urological infections. Due to the almost complete renal excretion, AGs are considered equivalent to first-choice antibacterial agents in the eradication of urinary tract infections (1), and show high efficacy in the treatment of complicated conditions (4), including highly-resistant, nosocomially-acquired urinary tract infections (NAUTI). A comprehensive, recent study on hospital-acquired urinary tract infections in urology departments, analyzing the data from both the Pan-European Prevalence and the Pan Euro-Asian Prevalence studies, has shown that besides fluoroquinolones (adopted as first-choice antibacterial agents, in $35 \%$ of cases), AGs are among the most commonly prescribed drugs for treatment of NAUTI (15\% of cases), together with penicillins $(16 \%)$ and 3 rd generation cephalosporins (14\%) (5). In 2004, AGs were recommended, in combination with $\beta$-lactam antibiotics, for
$\mathbf{A}$

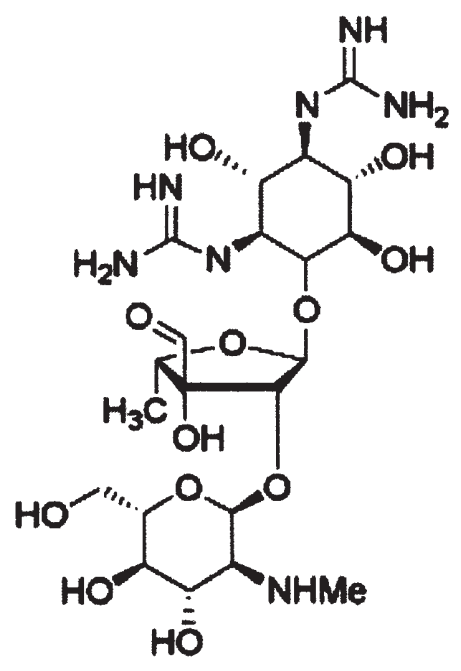<smiles>NC[C@H]1O[C@H](O[C@H]2[C@H](O)[C@H](O[C@@H]3[C@@H](O)[C@H](N)C[C@H](N)[C@H]3O[C@@H]3O[C@H](CO)[C@@H](O)[C@H](O)[C@H]3N)O[C@@H]2CO)[C@H](N)[C@@H](O)[C@H]1O</smiles>

C<smiles>[R2]NC([R1])[C@@H]1CC[C@@H](N)[C@@H](O[C@@H]2[C@H](N)C[C@H](N)[C@@H](O[C@@H]3OC[C@](C)(O)[C@H](NC)[C@H]3O)[C@@H]2O)O1</smiles>

Figure 1. Molecular structures of streptomycin (A), paromomycin (B) and gentamicin (C). R1 and R2 are substituents specific for gentamicin C1, C2 and C1a.

the treatment of category I acute bacterial prostatitis by the Chronic Prostatitis Collaborative Research Network, sponsored by the National Institute of Diabetes and Digestive and Kidney Diseases of the US National Institutes of Health (6).

Besides tuberculosis and complicated urinary tract infections, AGs are indicated for treatment of severe conditions such as complicated bone or soft tissue infection, septicemia, peritonitis and other severe intra-abdominal infections, endocarditis, and neonatal sepsis.

AGs are usually active against common Gram-negative uropathogens like E. coli, but also against specific Grampositive cocci, like Staphylococcus epidermidis. Enterococcus 


\section{CGUCACACC II I IIIII A}

Figure 2. Sequence of the E. coli decoding region A site. The adenine residue in position 1408 (base-paired with A1493) is highlighted.

faecalis, a very common Gram-positive uropathogen, is resistant to AGs in $\leq 35 \%$ of hospital-based infections, but is efficiently eradicated by aminoglycoside- $\beta$ lactam combination therapy (7).

\section{Bactericidal action of aminoglycosides}

Aminoglycosides are multifunctional hydrophilic aminosugars that are in most cases linked to a deoxystreptamine ring, and possess several amino moieties which are usually protonated in a physiological milieu. This confers to AGs the properties of water-soluble weak bases at body $\mathrm{pH}$, showing significant affinity for nucleic acids, and in particular for the $16 \mathrm{~S}$ ribosomal RNA (rRNA), in the $30 \mathrm{~S}$ subunit of bacterial ribosomes.

$16 \mathrm{~S}$ rRNA is essential in selecting tRNAs and in catalyzing peptide bond formation by ribosomes in prokaryotic cells. It is known that high fidelity of translation is achieved by the ability to discriminate between conformational changes induced by binding of cognate or non-cognate tRNAs at the aminoacyl-tRNA-binding ' $A$ ' site, in the decoding region of the 16S rRNA. Aminoglycosides act as inhibitors of protein synthesis because they increase the error rate of translation by allowing erroneous pairing of non-cognate tRNAs. 2-deoxystreptamine aminoglycosides interact with the major groove of the $16 \mathrm{~S}$ rRNA helix H44. Two universally conserved adenine residues (A1492 and A1493) in the rRNA are directly involved in the decoding process during normal translation. In the native structure of the ribosome, these residues are stacked in the interior of helix 44 ('off' conformation). Binding of the tRNA to the A site causes the displacement of both adenines out from their stacked position ('on' conformation). This conformational change is necessary for the specific directional interaction between A1492/1493 and two of three base pairs formed by the codon-anticodon interaction. The N1 of these adenines interacts through a hydrogen bond with the $2^{\prime} \mathrm{OH}$ in the mRNA codon, in the first and second positions of the codon-anticodon triplet. In this context, the pairing between cognate mRNAs and tRNAs ensures appropriate interaction between the codon and the ribosomal A site, whereas near-cognate tRNA and mRNA interactions distort the structure of the codon-anticodon helix and disrupts $\mathrm{N} 1-\mathrm{OH}$ contacts (8).

AGs like gentamicin and paromomycin (PM) (Fig. 1) can bind the major groove of rRNA within a pocket generated by Adenines 1408 and 1493 (Fig. 2), and a single bulged adenine (A1492). Fig. 3 models the binding of paromomycin to the A-site of 16S rRNA. An essential structural element for PM and neomycin-class AG binding to $16 \mathrm{~S}$ rRNA is the neamine core, a two-ring system comprising a 2-deoxystreptamine ring glycosylated at position 4 by a six-membered aminosugar of the glycopyranoside series (Fig. 1). Binding of PM results in the stabilization of adenines 1408, 1492 and 1493, and in the flipping-out of the latter two; this can reduce the energetic cost of codon-anticodon interactions, allowing binding of

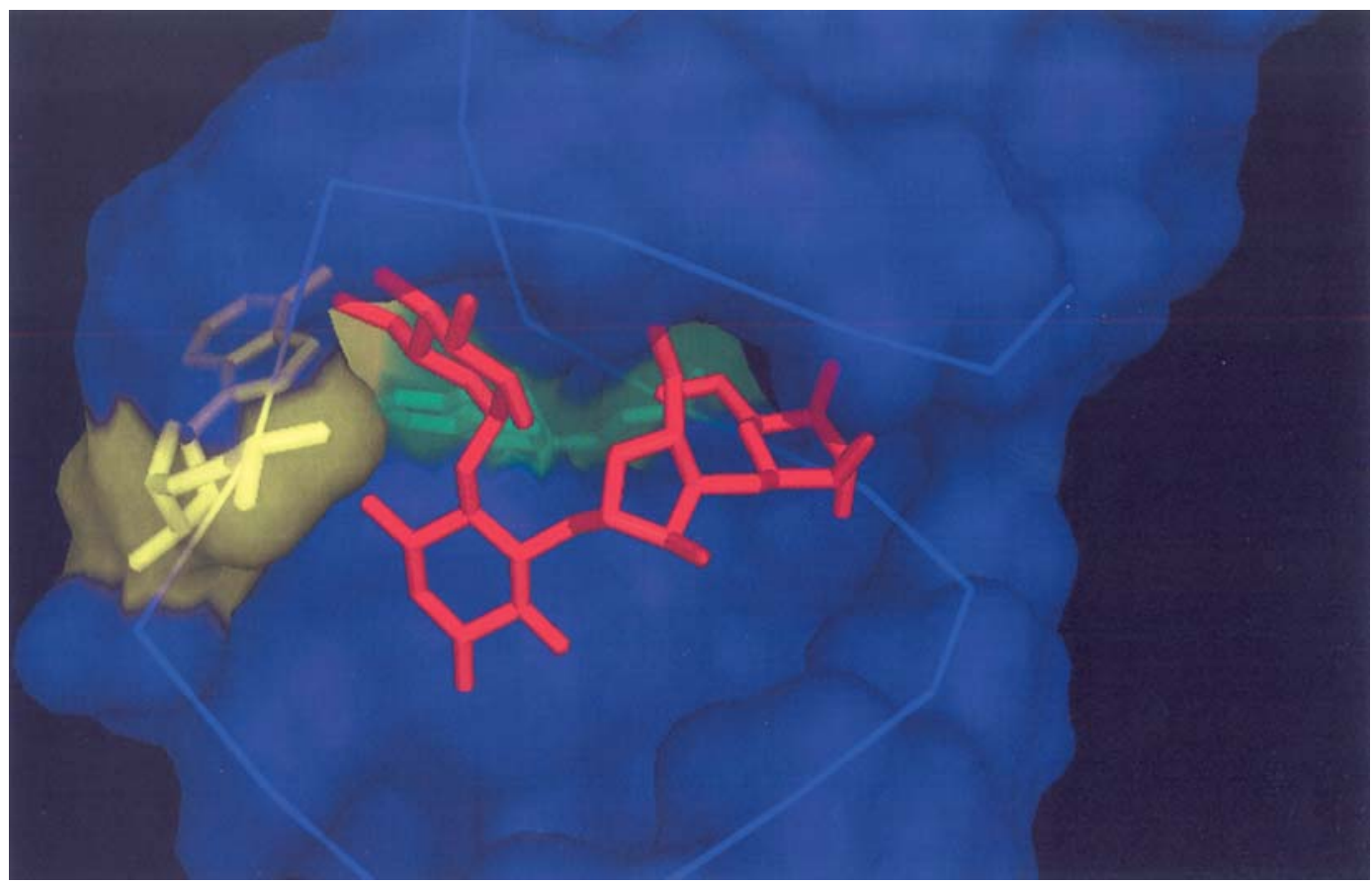

Figure 3. Model of the binding pocket of paromomycin in the A site of bacterial 16S rRNA. The Connolly surface of the RNA is represented in blue. Paromomycin is shown in red. Adenine 1408 and 1493 residues, creating a pocket for the binding of the aminoglycoside, are shown in green and in yellow, respectively. This picture was generated with the PyMol software (http://pymol.org), based on the deposited structure (PDB ID: 1PBR) of the paromomycinrRNA complex (9). 
near-cognate or non-cognate tRNAs and causing in turn the mistranslation of mRNA (9). For the implications in ototoxicity, it is necessary to underline that the C1409.G1491 base pair is essential for the antibacterial activity of some aminoglycosides, since mutation or methylation of G1491 was found by Guan et al to generate bacterial resistance to these antibiotics (10).

\section{Hearing loss induced by aminoglycosides}

Aminoglycosides are ototoxic when administered systemically or locally, and affect initially high frequency hearing beyond the range of normal speech. Thus, high frequency hearing loss can be detected by audiometric testing, which can be utilized to monitor AG-induced ototoxicity.

Renal impairment and pre-existing hearing loss can increase the risk of toxicity of these antibiotics to the inner ear. Moreover, the cumulative dose, the duration of treatment and repeated courses of therapy are critical determinants of toxicity at the level both of the kidneys and of the inner ear. As far as topical toxicity is concerned, it is known that AGs must not be prescribed for local administration in the presence of lesions of the tympanic membrane, because they can be readily absorbed through the fenestra rotunda, and can rapidly exert their toxic activity at the cochlear and vestibular levels.

Depending on their molecular structure, AG antibiotics are characterized by enhanced tropism and selectivity for different districts of the inner ear (11). Streptomycin (Fig. 1) targets preferentially the vestibular portion of the inner ear. Gentamicin, one of the most used AGs (Fig. 1), is also prevalently vestibulotoxic in patients. Although vertigo and balance problems tend to be only temporary due to subsequent compensation, impairment of the vestibular function can persist and manifest through difficulty of walking in the dark, or through the occurrence of the Dandy syndrome. In about $4-15 \%$ of patients treated with $1 \mathrm{~g} /$ day streptomycin for more than 7 days, hearing loss can also occur after a short latency (7-10 days). The condition can worsen if treatment is extended for longer periods.

Neomycin is characterized by a more marked cochleotoxic effect. Large doses of this drug, administered orally or locally for pre-surgery colon irrigation, can be absorbed, especially in the presence of ulcers and lesions within the GI mucosa. Similarly, kanamycin, amikacin, tobramycin and netilmycin (probably the least ototoxic members of the AG family) are prevalently cochleotoxic (11). Viomycin appears to be both cochleotoxic and vestibulotoxic.

Although hair cells in cristae, maculae or in the organ of Corti are the primary targets of AGs (12), an interesting study has demonstrated that cells in the stria vascularis, rich in mitochondria and responsible for the maintenance of the high potassium concentration of the endolymph $(\sim 157 \mathrm{mM}$, generating a positive $80-90 \mathrm{mV}$ potential relative to the perilymph) (13), are targets of AGs like amikacin (14). However, whereas the damage at the hair cell level can be permanent, stria vascularis, if affected, is only transiently affected by AGs (15).

It is now evident that outer hair cells are more sensitive to ototoxic injury than are inner hair cells. Aminoglycosides are transported into hair cells, can be detected in perilymph 60-90 min after systemic administration, and remain detectable in hair cells up to 4 months after exposure. In explants of inner ears subjected to aminoglycosides or other ototoxic drugs, a variety of reactive oxygen species can be detected, including both oxygen and nitrogen free radicals. Free radical damage to hair cells upon AG administration was shown to cause ototoxicity in a variety of in vitro and in vivo models (12). Recent findings by the Schacht group suggest that disruption of the actin cytoskeleton induced by kanamycin in cochlear sensory cells is mediated by Rac1 activation and the formation of superoxide by NADPH oxidase. Free radicals may in turn affect the RhoA/p140mDia gene pathway regulating actin, and affect cell survival and apoptotic pathways, ultimately resulting in loss of hair cells (16).

\section{Mutations predisposing to non-syndromic hearing loss (NSHL) and aminoglycoside ototoxicity}

Hearing loss can be caused by a variety of factors, and can be sporadic, genetically transmitted or drug-induced. Genetically transmitted hearing loss can be syndromic, i.e., combined with other conditions, thus creating a complex picture of pathologic alterations, or non-syndromic. In non-syndromic forms, which occur in about $70 \%$ of cases of hereditary hearing loss, deafness can be inherited by mitochondrial transmission, i.e., via the mother lineage.

A number of different genomic loci have been identified to be responsible for NSHL (reviewed in refs. 17,18). The most representative is DFNB1, responsible for over $50 \%$ of cases of prelingual deafness. This locus contains the Gap Junction Beta 2 (GJB2) gene, encoding connexin 26, a 26-kDa, 208-AA protein (19). Within gap junction plaques, connexins associate into hexamers, the connexons, forming juxtaposed intercellular channels connecting the cytosol of adjacent cells, allowing the exchange of ions and small molecules below a molecular weight of about $1 \mathrm{kDa}$. DFNB1 on chromosome 13 is a major locus for recessive deafness in Mediterranean affected families, and GJB2 is usually mutated in these pedigrees (20).

Mutations in the mitochondrial genome linked to hearing loss can be inherited or acquired, heteroplasmic or homoplasmic (reviewed in refs. 21,22). The most representative deafness-linked mitochondrial mutations - at the level of five distinct genes - are: i) A7445G (segregating with palmoplantar keratoderma in some individuals), 7472insC (associated with ataxia and/or focal myoclonus in some individuals), T7510C and T7511C, located in or adjacent to the tRNA $^{\text {Ser(UCN) }}$ gene; ii) $\mathrm{A} 3243 \mathrm{G}$, in the tRNA ${ }^{\text {leu(UUR) }}$ gene (can be associated with diabetes mellitus; 23,24); iii) A8344G, in the tRNA ${ }^{\text {lys }}$ gene; iv) T8993G, in the ATPase 6 gene; v) A827G, 961insC, 961delT/T961G (both associated in some cases with aminoglycoside ototoxicity), T1005C, T1095C, A1116G, T1291C, C1494T and A1555G, in the gene encoding the 12S rRNA (25-29). Interestingly, whereas in most cases of NSHL mutations are homoplasmic, all mutations linked to syndromic hearing impairment have been shown so far to be heteroplasmic.

The homoplasmic A1555G substitution, located in the mitochondrial $12 \mathrm{~S}$ ribosomal RNA gene, was the first mutation 
to be associated with non-syndromic hearing loss, but not with vestibular alterations. It was identified by Prezant and coworkers in the mother lineage of an Arab-Israeli pedigree, whose members showed the appearance of severe to profound sensorineural deafness during childhood or adulthood (30). In carriers of this mutation hearing loss can arise spontaneously or following exposure to aminoglycoside antibiotics (30). In A1555G individuals, spontaneous deafness does not occur in all carriers of this mutation, and intra- or inter-pedigree penetrance variations are likely due to the contribution of nuclear modifier genes. MTO1 is a gene responsible for tRNA hypermodification, a process that conditions the efficiency and accuracy of codon-anticodon interaction. This gene was shown to be responsible for the phenotypic expression of spontaneous deafness associated with the A1555G mutation (31).

A $1555 \mathrm{G}$ is expected to be the most prevalent deafnesslinked mitochondrial mutation worldwide, having been identified in both Caucasians and Asians as part of different, unrelated haplogroups $(21,23,24)$. It appears that the mutation is more frequent in Asian populations (32), as the majority of episodes of ototoxicity have been described in Chinese and other Asian patients. However, it has to be taken into account that aminoglycosides are more commonly prescribed - out of hospital settings - in the Asian continent than in Western European countries: this might mask an unsuspected higher prevalence of the mutation in Europe and in European-descent populations. It is however clear that the higher prevalence of cases of ototoxicity in Asia is not due to variations in the pharmacokinetic profile of AG antibiotics, which was shown to be very similar in different populations (33). In the Mediterranean area, a relatively high prevalence of the A1555G mutation has been described. A study on Spanish pedigrees reveals that the mutation accounts for about 15-20\% of analyzed familial cases of non-syndromic hearing loss, irrespective of their mode of inheritance and age of onset (28). Moreover, a phylogenetic analysis performed on 50 unrelated Spanish families showed that the A1555G substitution may have been generated by over 30 independent mutational events, occurring in mitochondrial DNA (mtDNA) haplo-groups that are found in all European populations. Indeed, the same mutation has been identified in Italian families or in individuals with Italian descent affected by aminoglycoside-induced or spontaneous non-syndromic hearing loss $(27,34,35)$. This suggests that the mutation might show a significant prevalence in patients affected by mitochondrial hearing loss in Italy, together with the less frequent T1095C and A7445 mutations, also described in Italian individuals and families $(35,36)$.

As previously mentioned, the A1555G mutation is located in the region of mitochondrial DNA encoding for the $12 \mathrm{~S}$ rRNA, a component of the $28 \mathrm{~S}$ subunit of the mitochondrial ribosome. It has been shown that the $\mathrm{G}$ variant of this mutation can increase the affinity of aminoglycosides for the 12S rRNA (37). The substitution of the adenine 1555 with a guanine would allow the pairing with a corresponding cytosine 1494, thus modifying the secondary structure of the RNA, at the level of the stem of a specific stem-loop structure (Fig. 4). Thus, the C1494-G1555 base pair in mitochondrial 12S rRNA would mimic a critical C1409-G1491 pair, important for the binding of specific AGs to the prokaryotic 16S rRNA in the decoding region of the bacterial ribosome. In this way, the binding of AGs to the 12S rRNA would be facilitated by the conformational changes generated by the A1555G mutation. This interaction is considered to be ultimately responsible for the destruction of mitochondria and for the consequent damage to hair cells at the cochlear level (38).

An additional mutation, C1494T, identified in the mtDNA of a Chinese family, generates the A1555-T1494 base pair at the same position (37). This mutation predisposes to aminoglycoside-induced hearing loss, thus supporting a specific role of this specific base pair as target of the toxic activity of AGs. Fig. 4 shows the similarity between the highly conserved aminoglycoside-binding regions of $16 \mathrm{~S}$ bacterial and $12 \mathrm{~S}$ mitochondrial rRNAs, in the presence of the A $1555 \mathrm{G}$ or C1494T mutations.

\section{Prevention and modulation of aminoglycoside-induced ototoxicity}

The findings described above imply that a proportion of patients with aminoglycoside-induced ototoxicity harbor mutations in the 12S rRNA gene. Drug-induced hearing loss in these individuals could be easily prevented by investigation of family history and by mitochondrial DNA analysis. However, when administered at high doses or for prolonged periods aminoglycosides can be toxic to the inner ear in patients who do not bear known predisposing mutations. In these cases, toxicity has been in most cases ascribed to inner ear cell damage generated by oxygen free radicals and other highly reactive molecules. In recent years, a number of strategies have been identified and studied in vitro and in vivo for the biochemical modulation and the chemoprevention of AG ototoxicity. Most strategies are based on the administration of antioxidants and other compounds that may counteract the molecular and cellular damage caused by reactive oxygen species. In the following section, a review of some of these strategies is provided.

\section{Drug/compound: Fosfomycin}

Proposed mechanism of action. Fosfomycin inhibits the accumulation of the AG dibekacin in the kidney, and reduces its concentration in the kidney and in the serum. Reduction of serum levels decreases the amount of AG delivered to the inner ear.

Experimental evidence. Rats treated with $50 \mathrm{mg} / \mathrm{kg}$ dibekacin combined with $500 \mathrm{mg} / \mathrm{kg}$ fosfomycin show markedly lower levels of kidney and serum AG than animals receiving dibekacin alone (39).

\section{Drug/compound: Glutathione}

Proposed mechanism of action. The damaging effects of the aminoglycoside amikacin may be caused by a metabolite of the drug. Glutathione acts as a source of cysteine, useful for the metabolic detoxification of the metabolite.

Experimental evidence. Guinea pigs treated with glutathione in combination with amikacin show reduced damage to the organ of Corti, compared to amikacin-only controls (40). In the Garetz report (41), Guinea pigs were injected with $100 \mathrm{mg}$ / $\mathrm{kg} /$ day gentamicin. A group of animals received glutathione by gastric gavage immediately prior to each gentamicin 


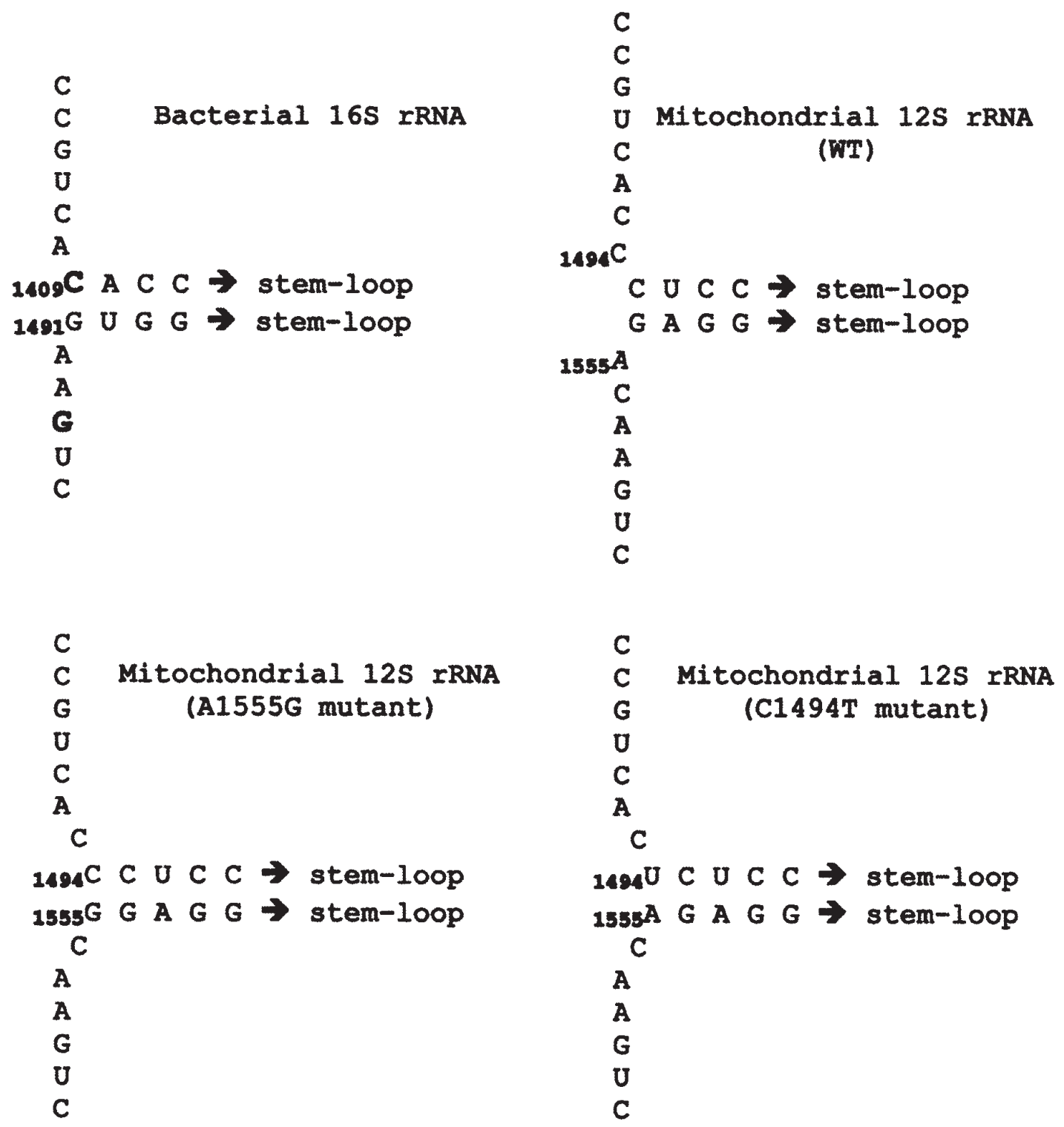

Figure 4. Sequence similarity between bacterial (E. coli) $16 \mathrm{~S}$ (top, left) and human mitochondrial 12S (top, right) rRNAs. Changes in the secondary structure of the 12S rRNA are shown as a consequence of the A1555G (bottom, left) and C1494T (bottom, right) mutations. Highlighted bases in the 16S rRNA are critical for binding of aminoglycosides. Base-pairing of the upper stem of the A site is not shown.

injection. Auditory brainstem responses revealed a progressive gentamicin-induced hearing loss reaching a 30-40 dB threshold shift at $2 \mathrm{kHz}$, approximately $60 \mathrm{~dB}$ at $8 \mathrm{kHz}$ and $80 \mathrm{~dB}$ at $18 \mathrm{kHz}$. Glutathione significantly attenuated the final threshold shifts by 20-40 dB. Morphological evaluation confirmed hair cell loss after gentamicin treatment and protection by glutathione.

Drug/compound: $\alpha$-lipoic acid

Proposed mechanism of action. The compound can prevent the cochlear toxicity following topic administration of neomycin to the round window. $\alpha$-lipoic acid is a free radical scavenger, protecting the inner ear from damage induced by reactive oxygen species. Among these, superoxide is generated by drugs (including AGs) that cause a deficiency of mitochondrial complex I (38).

Experimental evidence. Animals were implanted with osmotic pumps delivering to the round window a solution containing
$5 \%$ neomycin alone or in combination with $50 \mathrm{mg} / \mathrm{ml}$ $\alpha$-lipoic acid. Animals treated with combination therapy experienced a decreased rate of deterioration of auditory sensitivity (42).

Drug/compound: Leupeptin (calpain inhibitor); BAF (caspase inhibitor)

Proposed mechanism of action. Inner ear hair cell death following AG exposure was shown to be due to apoptosis. Apoptosis is mediated by caspases and calpain. Caspase and calpain inhibitors may prevent hair cell death by specific enzymatic inhibition.

Experimental evidence. TUNEL-labeled cells showing apoptotic DNA fragmentation were observed in the region of hair cells exposed to $0.2 \mathrm{mM}$ neomycin, in mouse cochlear explant cultures. The caspase inhibitor BAF $(200 \mu \mathrm{M})$ promoted the survival of $50 \%$ of cells. The compound was not protective when higher AG doses were used $(1 \mathrm{mM})$. Leupeptin 
$(10 \mu \mathrm{g} / \mathrm{ml})$ was significantly more efficient than BAF in protecting hair cells from AG-induced apoptosis $(43,44)$.

\section{Drug/compound: Dexamethasone}

Proposed mechanism of action. Reactive oxygen species have shown to be generated by a metabolic by-product of gentamicin. These species generate cell/tissue damage by lipid peroxidation and DNA/protein damage. Dexamethasone can counteract ROS formation by a mechanism that still has to be fully studied.

Experimental evidence. Kanamycin (400 mg/kg) combined with ethacrynic acid $(40 \mathrm{mg} / \mathrm{ml})$ was used to induce hair cell death and a severe hearing loss (threshold shift: 80-100 dBSPL) in Guinea pigs. Dexamethasone $(1 \mathrm{ng} / \mathrm{ml})$, delivered by intracochlear administration with microcannulation osmotic pumps, enhanced the auditory brainstem response (ABR), and at higher doses $(10 \mathrm{ng} / \mathrm{ml})$ attenuated the damage to outer hair cells (45).

Drug/compound: iron chelators [Deferoxamine (DFO), 2,3dihydroxybenzoate $(\mathrm{DHB})]$

Proposed mechanism of action. The ototoxic effects of gentamicin require an active metabolite form of the drug. Metabolic activation proceeds via the formation of a redoxactive irongentamicin complex that catalyzes the production of oxygenfree radicals. Agents that can prevent the formation of this complex by titrating intracellular iron show protective activity against cochlear and vestibular AG toxicity. Chu and coworkers (46) have shown that DHB attenuates AG-induced ototoxicity by enhancing the expression of the $\mathrm{Na}-\mathrm{K}-2 \mathrm{Cl}$ cotransporter-1 (NKCC1) in the marginal cells of the stria vascularis and in type II fibrocytes in the inferior portion of the spiral ligament. $\mathrm{NKCC} 1$ is responsible for the basolateral uptake of potassium ions into stria vascularis cells from the interatrial space. Potassium is then apically secreted by the same cells into the endolymph, thus preserving the unique $\mathrm{K}^{+}$ concentration in this fluid. AG-generated free radicals are responsible for the destruction of NKCC1.

Experimental evidence. In Guinea pigs, DHB (100 mg/kg/day) attenuated a gentamicin-induced threshold shift of about $60 \mathrm{~dB}$ to approximately $10 \mathrm{~dB}$, whereas DFO significantly reduced the threshold to about $40 \mathrm{~dB}$ at $18 \mathrm{kHz}$ (47). In addition, DHB was efficient in fully protecting the vestibular compartment from streptomycin-induced nystagmus. Moreover, DHB treatment affects molecular survival mechanisms in hair cells (48). In the Conlon study, animals receiving neomycin experienced elevation of elicited compound action potential thresholds (CAP) of $37.5 \mathrm{~dB}$ at 2 or $4 \mathrm{kHz}$, and of $38.8 \mathrm{~dB}$ at $8 \mathrm{kHz}$. Animals treated with neomycin and deferoxamine $(150 \mathrm{mg} / \mathrm{kg}$ twice daily) maintained CAPelicited thresholds at all tested frequencies (49). In the study of Sinswat et al (50), Guinea pigs were treated with 120 or $135 \mathrm{mg} / \mathrm{kg}$ gentamicin. Intraperitoneal administration of DHB $(100 \mathrm{mg} / \mathrm{kg}$ ) attenuated the progressive hearing loss induced by gentamicin (threshold shifts: $60 \mathrm{~dB}$ at $18 \mathrm{kHz}$ ) to less than $30 \mathrm{~dB}$, and reduced the loss of hair cells.

\section{Drug/compound: Salicylates}

Proposed mechanism of action. As described above, AGs catalyze the formation of free radicals through an iron- dependent reaction. Salicylates act as iron chelators and antioxidants, and protect against hearing loss. In addition, salycilates (and DHB, see previous paragraph) can promote the translocation of nuclear factor (NF)- $\mathrm{\kappa B}$ into the nuclei of outer hair cells, and thus prevent hair cell death by induction of a specific survival pathway, as described by Jiang et al (51).

Experimental evidence. Combination of gentamicin and salicylates protected from an auditory threshold shift $(>60$ $20 \mathrm{~dB}$ ) induced by gentamicin alone. Morphological assessment showed protection of inner hair cells. The treatment did not change gentamicin serum levels and was not detrimental to the antibacterial activity of the drug (52).

Clinical evidence. A prospective, randomized, placebocontrolled, double-blind clinical trial was conducted on a total of 195 patients, aged 18-65, receiving total average doses of about $980 \mathrm{mg}$ gentamicin. Aspirin was administered in thricedaily doses of $1000 \mathrm{mg}$ for 14 days. The total incidence of hearing loss decreased from $13 \%$ in the gentamicin-only group to $3 \%$ in the gentamicin/aspirin group; moderate hearing losses (15-25 dB) decreased from 11 to $1 \%$, respectively. Five patients in the aspirin group reported gastric discomfort, and three were excluded from the trial for gastric bleeding $(53,54)$.

\section{Drug/compound: Tanshinone [Saliae miltiorrhizae (Danshen) extract]}

Proposed mechanism of action. The diterpene quinone and phenolic acid derivatives contained in S. miltiorrhizae (Danshen) have antioxidant properties in vitro and in vivo, and can act as scavengers of free radicals generated by metabolic activation of aminoglycosides.

Experimental evidence. In vitro, Danshen inhibited gentamicincatalyzed formation of superoxide and hydroxy radicals, as well as gentamicin-induced lipid peroxidation. In CBA mice treated with $700 \mathrm{mg} / \mathrm{kg}$ gentamicin and various doses of Danshen, attenuation of AG-induced auditory threshold shifts $(\sim 50 \mathrm{~dB})$ was documented (55).

\section{Drug/compound: 4-methylcatechol}

Proposed mechanism of action. AGs are toxic on spiral ganglion neurons through a direct effect, but also indirectly, by inducing the loss of neurotropic factors. 4-methylcatechol protects spiral ganglion neurons by inducing the synthesis of nerve growth factor.

Experimental evidence. The number of surviving spiral ganglion neurons was significantly higher in mice treated with gentamicin in combination with 4-methylcatechol than in animals receiving the aminoglycoside alone (56).

\section{Drug/compound: Concanavalin A}

Proposed mechanism of action. Concanavalin A interferes with the uptake of gentamicin into hair cells in vitro.

Experimental evidence. Postnatal rat cochlear explant cultures were exposed to gentamicin with or without $100 \mathrm{mM}$ concanavalin A. Significant protection was observed by confocal microsopy only in hair cells co-exposed to concanavalin (57).

\section{Drug/compound: Trimetazidine}

Proposed mechanism of action. Protection from free-radical injury. 
Experimental evidence. Swiss mice received gentamicin for 30 days, with or without trimetazidine. Auditory threshold shifts were measured by evoked brain stem response, and were found to be significantly different from controls in animals receiving the AG. Conversely, threshold shifts in the trimetazidine-gentamicin group were not significantly different from control animals that did not receive the AG (58).

\section{Drug/compound: Flavonoid fraction (FF) of Drynaria fortunei}

Experimental evidence. Guinea pigs were treated with $100 \mathrm{mg} /$ $\mathrm{kg}$ day gentamicin for two weeks. A group of animals was pretreated with $10 \mathrm{mg} / \mathrm{kg} /$ day flavonoid fraction. The gentamicin-only group showed a higher ABR threshold burst: 90 , 92 and $72 \mathrm{~dB}$ at 2,8 and $32 \mathrm{kHz}$, respectively, when compared to the gentamicin+FF group (30, 37 and $38 \mathrm{~dB})(59)$.

Drug/compound: basic fibroblast growth factor ( $F G F-2)$

Experimental evidence. In neonatal rat organ of Corti cultures inner hair cell survival and outer hair cell destruction $(50 \%$ at $35 \mathrm{mM}$ neomycin) was observed after neomycin administration at micromolar concentrations. A protective effect was observed when the same cultures were pretreated and co-exposed to

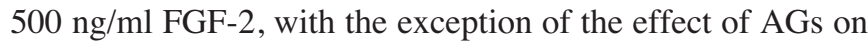
disorganization of inner cell stereocilia (60).

Drug/compound: Transforming growth factor alpha (TGF- $\alpha)$ Proposed mechanism of action. A rise in cell calcium levels in hair cells of organ of Corti explants correlates with the protection from aminoglycoside toxicity by exposure to TGF- $\alpha$.

Experimental evidence. $500 \mathrm{ng} / \mathrm{ml}$ TGF- $\alpha$ increases by 5 -fold the intracellular calcium concentration in hair cells (61).

\section{Drug/compound: $M 40403$}

Proposed mechanism of action. M40403 is a manganese-based molecule that mimics the activity of superoxide dismutase. This molecule can protect inner hair cells from AG-induced generation of reactive oxygen species by facilitating the metabolism of superoxide radicals.

Experimental evidence. Inner and outer hair cells within cochlear cultures from neonatal C57BL/10J mice were significantly protected by low micromolar doses M30403 against toxicity induced by gentamicin. However, 30 micro-molar M30403 showed significant toxicity on the same cells. Interestingly, the protective effect was not observed upon exposure to ototoxic cisplatin (62).

\section{Drug/compound: Argon}

Proposed mechanism of action. Argon can protect neuronal cells from hypoxic damage.

Experimental evidence. Organotypic newborn rat organs of Corti cultures exposed to Argon-oxygen mixtures $\left(21 \% \mathrm{O}_{2}\right.$, $5 \% \mathrm{CO}_{2}, 74 \% \mathrm{Ar}$ ) were protected against the toxic activity of 5-40 micromolar gentamicin or 7-25 micromolar cisplatin (63).

\section{Drug/compound: D-methionine}

Proposed mechanism of action. D-methionine is an amino-acid showing anti-oxidant and iron-chelating properties and protects from AG-induced generation of free radicals (see above).
Experimental evidence. Threshold shifts induced by $120 \mathrm{mg} / \mathrm{kg}$ gentamicin $(40 \mathrm{~dB}$ at $3 \mathrm{kHz} ; 50-60 \mathrm{~dB}$ at $18 \mathrm{kHz}$ ) were significantly reduced at all frequencies when $\mathrm{D}$-methionine was co-administered at the twice-daily dose of $200 \mathrm{mg} / \mathrm{kg}$. D-methionine $(30 \mathrm{mM})$ also was effective in reducing the gentamicin-induced generation of free radicals in EBVtransformed lymphoblastoid cells (64).

\section{Drug/compound: fusion protein TAT-FNK}

Proposed mechanism of action. FNK was constructed by introducing amino-acid substitutions into the Bcl-XL antiapoptotic protein, creating a mutant with a gain-of-function phenotype, showing higher anti-apoptotic activity to protect cultured cells from death induced by various stimuli, including oxidative stress and AG-induced toxicity to hair cells of the inner ear. When fused to the TAT protein transduction domain of the HIV/TAT protein, FNK is readily introduced into target cells, where it can exert its protective function against AGinduced damage.

Experimental evidence. TAT-FNK attenuated cochlear damage induced by $200-400 \mathrm{mg} / \mathrm{kg}$ kanamycin, administered in combination with ethacrynic acid to Guinea pigs. Auditory brainstem response threshold shifts induced by kanamycin were markedly attenuated in animals treated with TAT-FNK. A protective activity by TAT-FNK against AG-induced apoptosis was also demonstrated by the reduction of cleaved poly-(ADP-ribose) polymerase in hair cells 8 hours after AG administration (65).

Drug/compound: MK801 (N-methyl-D-Aspartate antagonist); neurotrophin 3 (NT3)

Proposed mechanism of action. In the peripheral auditory system, the excitatory neurotransmitter glutamate acts by binding the N-methyl-D-Aspartate (NMDA) receptor, located on the terminals of the spiral ganglion neurons. Cell death by excitotoxicity is caused by a calcium overload induced by overstimulated NMDA receptors. AGs mimic the effect of polyamines on the NMDA receptor and cause excitotoxicity. The NMDA antagonist MK801 can counteract this effect. NT3 cooperates by promoting survival of auditory neurons.

Experimental evidence. In amikacin-treated $(300 \mathrm{mM}$ administered by osmotic pumps) Guinea pigs, combined treatment of MK801 (1 mg/kg/day X 3, i.p.) and NT3 (300 ng/h for $24 \mathrm{~h}$ ) caused partial protection of hair cells ( $\geq 50 \%$ cell survival vs. $0 \%$ in amikacin-only group) and complete protection of spiral ganglion neurons (103\% survival vs. $10.4 \%$ in amikacin-only group); moreover, auditory brainstem response threshold shifts were significantly decreased, and remained within $20 \mathrm{~dB}$ of normal values (66-68).

\section{Drug/compound: non-ototoxic doses of the aminoglycoside amikacin}

Proposed mechanism of action. As described in previous sections, aminoglycosides can chelate iron and form ototoxic metabolites; these complexes generate free oxygen radicals that in turn cause hair cell damage. A subtoxic dose of AG may cause a gradual 'preparation' of these cells for 'selfdefence', likely by increasing the levels of anti-free radical agents like glutathione. It should be remarked that a pretreatment with low dose aminoglycosides is possible only in laboratory conditions. Patients diagnosed with infectious 
diseases cannot be treated with ineffective low doses of antibiotics, because therapy has to commence immediately. Furthermore, a low-dose treatment with aminoglycosides would increase the bacterial resistance, thus being counterproductive to an effective therapy.

Experimental evidence. When a group of Guinea pigs treated with $400 \mathrm{mg} / \mathrm{kg}$ amikacin for 12 days was compared to animals pre-treated with low-doses of the drug ( $20 \mathrm{mg} / \mathrm{kg}$ for 30 days followed by $400 \mathrm{mg} / \mathrm{kg}$ for 12 days), the conservation of outer hair cells was found to be significantly higher in the latter group, especially in the more basal turns of the cochlea (69).

\section{Drug/compound: $\alpha$-phenyl-tert-butyl-nitrone (PBN)}

Proposed mechanism of action. PBN acts as a scavenger of free radicals generated by metabolic activation of AGs.

Experimental evidence. Guinea pigs were monitored for compound action potentials thresholds. Animals were subjectd to treatment with $60 \mathrm{mg} / \mathrm{kg} \mathrm{PBN}$, followed by application of $\mathrm{PBN}$ to the round window membrane, and by local application of AG. This protocol significantly decreased the compound action potential threshold sensitivity loss observed in the presence of the aminoglycoside alone (70).

\section{Dose and regimen optimization}

Due to their pharmacokinetic/pharmacodynamic properties, aminoglycosides may be administered using flexible dosing regimens. In addition to traditional medium-term, twice/thricedaily parenteral administration, single-daily dosing (SDD) or switch-therapy regimens have been adopted as effective therapeutic strategies.

In particular, due to the long urinary half-life of AGs (>100 h, with a 3-compartment modeled distribution), singledaily dosing regimens have been shown to be effective in the treatment of severe urological conditions like pyelonephritis (7). Since over a decade, a number of preclinical and clinical studies have been performed to compare the effectiveness and tolerability of SDD vs. multiple daily dosing (MDD) of AGs. Although not recommended for pediatric patients, SDD is believed to evoke increased bactericidal effects due to higher peak serum levels, decreased emergence of resistant organisms, longer post-antibiotic effect and increased time lag for resolution of adaptive resistance. Animal studies and a metaanalysis of clinical trials have shown that single daily dosing (e.g. $5-7 \mathrm{mg} / \mathrm{kg}$ every $24 \mathrm{~h}$ ) of AGs is less ototoxic than MDD (reviewed in ref. 7). However, other meta-analyses did not demonstrate differential toxicity between the two dosing regimens. A switch therapy regimen based on a single large daily dose of gentamicin $(10 \mathrm{mg} / \mathrm{kg})$, followed by twice-daily ciprofloxacin $(250 \mathrm{mg}$ ) for five days, has been compared to a multiple dose aminoglycoside-ciprofloxacin protocol in the context of a prospective, randomized controlled clinical trial which included 53 acute pyelonephritis patients. It was demonstrated that the former regimen induced ototoxicity in $17 \%$ of cases, whereas the latter showed a higher rate (30\%) of hearing impairment episodes (71). This was likely due to the combined effects of the single-dose strategy and the switch to a non-ototoxic antibacterial agent. Interestingly, the two regimens were equally effective in eradicating the causative agents of pyelonephritis.
It seems paradoxical that, although SDD induces higher peak serum concentrations, lower ototoxicity is observed when compared to MDD. However, it is known that inner ear uptake of AGs is a saturation process. According to Santucci and Krieger, with SDD inner ear cells are saturated only once, whereas with MDD saturation occurs 2-3 times within $24 \mathrm{~h}$ (7), thus increasing the cellular aminoglycoside load.

\section{Conclusions}

Damage caused by reactive oxygen species on hair cells was shown to be a general mechanism of AG ototoxicity. In genetically predisposed individuals, AGs target the mitochondrial $12 \mathrm{~S}$ ribosomal RNA, and likely disrupt mitochondria by affecting translation in a fashion resembling their bactericidal activity. However, in carriers of mutations at the mitochondrial level, free radicals may also play an important role in inner ear cell disruption, since generation of reactive oxygen species by defective oxidative phosphorylation may be due to mistranslation or altered expression of mitochondrial proteins involved in this process, caused in turn by inherited mutations in rRNA-encoding sequences.

An impressive amount of pharmacological and molecular strategies have been developed to counteract the toxic effect of aminoglycosides in the inner ear in non-genetically predisposed individuals. However, these data have been obtained in preclinical settings, and extensive clinical trials will be necessary to validate the above-described evidence, given that potentially protective drugs should be non-toxic to the patient by systemic administration, should not lower aminoglycoside serum levels, and should not interfere with the antibacterial activity of the drugs.

As summarized above, in a recent double-blind, placebocontrolled clinical trial it was shown that administration of acetylsalicylic acid can significantly attenuate the risk of gentamicin-induced hearing loss (53). Due to the convincing clinical and preclinical data, and to the fact that the proposed protocol is simple and cost-effective, aspirin is likely to be further investigated for adoption as an oto-protective agent in the clinical practice. In particular, it would be interesting to ascertain whether dose reduction may decrease the episodes of gastric intolerance without affecting the protective potential of the drug. However, the suggested dose of $3 \mathrm{~g} /$ day is currently authorized for treatment of rheuma patients ( $2-4 \mathrm{~g} / \mathrm{day})$; to decrease the incidence of episodes of gastric intolerance aspirin could be administered in the form of enteric-coated tablets, with particular caution on the potential adverse effects in sensitive individuals.

As shown in the articles and reviews cited herein, extensive research has been performed to characterize the mechanisms whereby aminoglycosides exert their ototoxic activity, and to discover new agents or new dosing regimens aimed at minimizing these effects. If correctly validated and applied, some of the evidence will surely be beneficial to patients and useful to clinicians, and will allow a more extensive and safer use of these old, yet potent antimicrobial agents.

\section{Acknowledgments}

This work was supported in part by Konpharma S.r.l., Italy. 


\section{References}

1. Vakulenko SB and Mobashery S: Versatility of aminoglycosides and prospects for their future. Clin Microbiol Rev 16: 430-450, 2003.

2. Smith DG and Waksman SA: Tuberculostatic and tuberculocidal properties of streptomycin. J Bacteriol 54: 253-261, 1947.

3. Di Perri G and Bonora S: Which agents should we use for the treatment of multidrug-resistant Mycobacterium tuberculosis? J Antimicrob Chemother 54: 593-602, 2004.

4. Fang GD, Brennen C, Wagener M, Swanson D, Hilf M, Zadecky L, De Vine J and Yu VL: Use of ciprofloxacin versus use of aminoglycosides for therapy of complicated urinary tract infection: prospective, randomized clinical and pharmacokinetic study. Antimicrob Agents Chemother 35: 1849-1855, 1991.

5. Bjerklund Johansen TE, Cek M, Naber KG, Stratchounski L, Svendsen MV and Tenk P: Hospital acquired urinary tract infections in urology departments: pathogens, susceptibility and use of antibiotics. Data from the PEP and the PEAP studies. Int J Antimicrob Agents 28S: S91-S107, 2006.

6. Schaeffer AJ; National Institute of Diabetes and Digestive and Kidney Diseases of the US National Institutes of Health: NIDDKsponsored chronic prostatitis collaborative research network (CPCRN) 5-year data and treatment guidelines for bacterial prostatitis. Int J Antimicrob Agents 24 (Suppl. 1): S49-S52, 2004.

7. Santucci RA and Krieger JN: Gentamicin for the practicing urologist: review of efficacy, single daily dosing and 'switch' therapy. J Urol 163: 1076-1084, 2000.

8. Yoshizawa S, Fourmy D and Puglisi JD: Recognition of the codon-anticodon helix by ribosomal RNA. Science 285: 1722-1725, 1999

9. Fourmy D, Recht MI and Puglisi JD: Binding of neomycin-class aminoglycoside antibiotics to the A-site of $16 \mathrm{~S}$ rRNA. J Mol Biol 277: 347-362, 1998.

10. Guan MX, Fischel-Ghodsian N and Attardi G: A biochemical basis for the inherited susceptibility to aminoglycoside ototoxicity. Hum Mol Genet 9: 1787-1793, 2000.

11. Selimoglu E: Aminoglycoside-induced ototoxicity. Curr Pharm Des 13: 119-126, 2007.

12. Forge A and Schacht J: Aminoglycoside antibiotics. Audiol Neurootol 5: 3-22, 2000.

13. Takeuchi S, Ando M and Kakigi A: Mechanism generating endocochlear potential: role played by intermediate cells in stria vascularis. Biophys J 79: 2572-2582, 2000

14. Kalkandelen S, Selimoglu E, Erdogan F, Ucuncu H and Altas E: Comparative cochlear toxicities of streptomycin, gentamicin, amikacin and netilmicin in Guinea-pigs. J Int Med Res 30: 406-412, 2002

15. Komune S, Ide M, Nakano T and Morimitsu T: Effects of kanamycin sulfate on cochlear potentials and potassium ion permeability through the cochlear partitions. ORL J Otorhinolaryngol Relat Spec 49: 9-16, 1987.

16. Jiang H, Sha SH and Schacht J: Rac/Rho pathway regulates actin depolymerization induced by aminoglycoside antibiotics. J Neurosci Res 83: 1544-1551, 2006.

17. Kelsell DP, Di WL and Houseman MJ: Connexin mutations in skin disease and hearing loss. Am J Hum Genet 68: 559-568, 2001.

18. Petit C, Levilliers J and Hardelin JP: Molecular genetics of hearing loss. Annu Rev Genet 35: 589-646, 2001.

19. Estivill X, Fortina P, Surrey S, Rabionet R, Melchionda S, D'Agruma L, Mansfield E, Rappaport E, Govea N, Mila M, Zelante L and Gasparini P: Connexin-26 mutations in sporadic and inherited sensorineural deafness. Lancet 351: 394-398, 1998.

20. Lefebvre PP and van De Water TR: Connexins, hearing and deafness: clinical aspects of mutations in the connexin 26 gene. Brain Res Brain Res Rev 32: 159-162, 2000.

21. Fischel-Ghodsian N: Mitochondrial deafness. Ear Hear 24: 303-313, 2003.

22. Van Camp G and Smith RJ: Maternally inherited hearing impairment. Clin Genet 57: 409-414, 2000.

23. Fischel-Ghodsian N: Mitochondrial mutations and hearing loss: paradigm for mitochondrial genetics. Am J Hum Genet 62: 15-19, 1998.

24. Fischel-Ghodsian N: Mitochondrial deafness mutations reviewed. Hum Mutat 13: 261-270, 1999.

25. Zhao H, Li R, Wang Q, Yan Q, Deng JH, Han D, Bai Y, Young WY and Guan MX: Maternally inherited amino-glycosideinduced and non-syndromic deafness is associated with the novel C1494T mutation in the mitochondrial 12S rRNA gene in a large Chinese family. Am J Hum Genet 74: 139-152, 2004.
26. Zhao L, Young WY, Li R, Wang Q, Qian Y and Guan MX: Clinical evaluation and sequence analysis of the complete mitochondrial genome of three Chinese patients with hearing impairment associated with the 12S rRNA T1095C mutation. Biochem Biophys Res Commun 325: 1503-1508, 2004.

27. Casano RA, Bykhovskaya Y, Johnson DF, Hamon M, Torricelli F, Bigozzi M and Fischel-Ghodsian N: Hearing loss due to the mitochondrial A1555G mutation in Italian families. Am J Med Genet 79: 388-391, 1998

28. Del Castillo FJ, Rodriguez-Ballesteros M, Martin Y, Arellano B, Gallo-Teran J, Morales-Angulo C, Ramirez-Camacho R, Cruz Tapia M, Solanellas J, Martinez-Conde A, Villamar M, Moreno-Pelayo MA, Moreno F and Del Castillo I: Heteroplasmy for the $1555 \mathrm{~A}>\mathrm{G}$ mutation in the mitochondrial $12 \mathrm{~S}$ rRNA gene in six Spanish families with non-syndromic hearing loss. J Med Genet 40: 632-636, 2003.

29. Li Z, Li R, Chen J, Liao Z, Zhu Y, Qian Y, Xiong S, HemanAckah S, Wu J, Choo DI and Guan M-X: Mutational analysis of the mitochondrial 12S rRNA gene in Chinese pediatric subjects with aminoglycoside-induced and non-syndromic hearing loss. Hum Genet 117: 9-15, 2005.

30. Prezant TR, Agapian JV, Bohlman MC, Bu X, Oztas S, Qiu WQ, Arnos KS, Cortopassi GA, Jaber L, Rotter JI, Shohat M and Fischel-Ghodsian N: Mitochondrial ribosomal RNA mutation associated with both antibiotic-induced and non-induced syndromic deafness. Nat Genet 4: 289-294, 1993.

31. Li X, Li R, Lin X and Guan MX: Isolation and characterization of the putative nuclear modifier gene MTO1 involved in the pathogenesis of deafness-associated mitochondrial $12 \mathrm{~S}$ rRNA A1555G mutation. J Biol Chem 277: 27256-27264, 2002.

32. Malik SG, Pieter N, Sudoyo H, Kadir A and Marzuki S: Prevalence of the mitochondrial DNA A1555G mutation in sensorineural deafness patients in island Southeast Asia. J Hum Genet 48: 480-483, 2003.

33. Jhee SS, Burm JP and Gill MA: Comparison of aminoglycoside pharmacokinetics in Asian, Hispanic, and Caucasian patients by using population pharmacokinetic methods. Antimicrob Agents Chemother 38: 2073-2077, 1994

34. Fischel-Ghodsian N, Prezant TR, Chaltraw WE, Wendt KA, Nelson RA, Arnos KS and Falk RE: Mitochondrial gene mutation is a significant predisposing factor in aminoglycoside ototoxicity. Am J Otolaryngol 18: 173-178, 1997.

35. Jacobs HT, Hutchin TP, Käppi T, Gillies G, Minkkinen K, Walker J, Thompson K, Rovio AT, Carella M, Melchionda S, Zelante L, Gasparini P, Pyykkö I, Shah ZH, Zeviani M and Mueller RF: Mitochondrial DNA mutations in patients with postlingual, non-syndromic hearing impairment. Eur J Hum Genet 13: 26-33, 2005 .

36. Tessa A, Giannotti A, Tieri L, Vilarinho L, Marotta G and Santorelli FM: Maternally inherited deafness associated with a T1095C mutation in the mDNA. Eur J Hum Genet 9: 147-149, 2001.

37. Zhao H, Young WY, Yan Q, Li R, Cao J, Wang Q, Li X, Peters JL, Han D and Guan MX: Functional characterization of the mitochondrial $12 \mathrm{~S}$ rRNA C1494T mutation associated with aminoglycoside-induced and non-syndromic hearing loss. Nucleic Acids Res 33: 1132-1139, 2005.

38. Hutchin T and Cortopassi G: Proposed molecular and cellular mechanism for aminoglycoside ototoxicity. Antimicrob Agents Chemother 38: 2517-2520, 1994.

39. Ohtani I, Ohtsuki K, Aikawa T, Sato Y, Anzai T and Ouchi J: Mechanism of protective effect of fosfomycin against aminoglycoside ototoxicity. Auris Nasus Larynx 11: 119-124, 1984.

40. Nishida I and Takumida M: Attenuation of aminoglycoside ototoxicity by glutathione. ORL J Otorhinolaryngol Relat Spec 58: 68-73, 1996.

41. Garetz SL, Altschuler RA and Schacht J: Attenuation of gentamicin ototoxicity by glutathione in the guinea pig in vivo. Hear Res 77: 81-87, 1994.

42. Conlon BJ and Smith DW: Topical aminoglycoside ototoxicity: attempting to protect the cochlea. Acta Otolaryngol 120: 596-599, 2000.

43. Momiyama J, Hashimoto T, Matsubara A, Futai K, Namba A and Shinkawa H: Leupeptin, a calpain inhibitor, protects inner ear hair cells from aminoglycoside ototoxicity. Tohoku J Exp Med 209: 89-97, 2006.

44. Ding D, Stracher A and Salvi RJ: Leupeptin protects cochlear and vestibular hair cells from gentamicin ototoxicity. Hear Res 164: 115-126, 2002 
45. Himeno C, Komeda M, Izumikawa M, Takemura K, Yagi M, Weiping Y, Doi T, Kuriyama H, Miller JM and Yamashita T: Intracochlear administration of dexamethasone attenuates aminoglycoside ototoxicity in the guinea pig. Hear Res 167: 61-70, 2002.

46. Chu HQ, Xiong H, Zhou XQ, Han F, Wu ZG, Zhang P, Huang XW and Cui YH: Aminoglycoside ototoxicity in three murine strains and effects on NKCC1 of stria vascularis. Chin Med J 119: 980-985, 2006.

47. Song BB, Sha SH and Schacht J: Iron chelators protect from aminoglycoside-induced cochleo- and vestibulo-toxicity. Free Radic Biol Med 25: 189-195, 1998.

48. Jiang H, Sha SH and Schacht J: NF-kappaB pathway protects cochlear hair cells from aminoglycoside-induced ototoxicity. J Neurosci Res 79: 644-651, 2005.

49. Conlon BJ, Perry BP and Smith DW: Attenuation of neomycin ototoxicity by iron chelation. Laryngoscope 108: 284-287, 1998.

50. Sinswat P, Wu WJ, Sha SH and Schacht J: Protection from ototoxicity of intraperitoneal gentamicin in guinea pig. Kidney Int 58: 2525-2532, 2000.

51. Jiang H, Sha SH and Schacht J: NF-kappaB pathway protects cochlear hair cells from aminoglycoside-induced ototoxicity. J Neurosci Res 79: 644-651, 2005.

52. Sha SH and Schacht J: Salicylate attenuates gentamicin-induced ototoxicity. Lab Invest 79: 807-813, 1999.

53. Sha S-H, Qiu J-H and Schacht J: Aspirin attenuates gentamicininduced hearing loss. N Engl J Med 354: 1856-1857, 2006.

54. Chen Y, Huang WG, Zha DJ, Qiu JH, Wang JL, Sha SH and Schacht J: Aspirin attenuates gentamicin ototoxicity: from the laboratory to the clinic. Hear Res 226: 178-182, 2007.

55. Wang AM, Sha SH, Lesniak W and Schacht J: Tanshinone (Salviae miltiorrhizae extract) preparations attenuate aminoglycoside-induced free radical formation in vitro and ototoxicity in vivo. Antimicrob Agents Chemother 47: 1836-1841, 2003.

56. Kimura N, Nishizaki K, Orita Y and Masuda Y: 4-methylcatechol, a potent inducer of nerve growth factor synthesis, protects spiral ganglion neurons from aminoglycoside ototoxicity - preliminary report. Acta Otolaryngol Suppl 540: 12-15, 1999.

57. Zheng JL and Gao WQ: Concanavalin A protects hair cells against gentamicin ototoxicity in rat cochlear explant cultures. J Neurobiol 39: 29-40, 1999.

58. Unal OF, Ghoreishi SM, Atas A, Akyurek N, Akyol G and Gursel B: Prevention of gentamicin induced ototoxicity by trimetazidine in animal model. Int J Pediatr Otorhinolaryngol 69: 193-199, 2005.

59. Long M, Smouha EE, Qiu D, Li F, Johnson F and Luft B: Flavonoid of Drynaria fortunei protects against gentamicin ototoxicity. Phytother Res 18: 609-614, 2004.
60. Low W, Dazert S, Baird A and Ryan AF: Basic fibroblast growth factor (FGF-2) protects rat cochlear hair cells in organotypical culture from aminoglycoside injury. J Cell Physiol 167: 443-450, 1996.

61. Staecker H, Dazert S, Malgrange B, Lefebvre PP, Ryan AF and van de Water TR: Transforming growth factor alpha treatment alters intracellular calcium levels in hair cells and protects them from ototoxic damage in vitro. Int J Dev Neurosci 15: 553-562, 1997.

62. McFadden SL, Ding D, Salvemini D and Salvi RJ: M40403, a superoxide dismutase mimetic, protects cochlear hair cells from gentamicin, but not cisplatin toxicity. Toxicol Appl Pharmacol 186: 46-54, 2003.

63. Yarin YM, Amarjargal N, Fuchs J, Haupt H, Mazurek B, Morozova SV and Gross J: Argon protects hypoxia-, cisplatinand gentamicin-exposed hair cells in the newborn rat's organ of Corti. Hear Res 201: 1-9, 2005.

64. Sha SH and Schacht J: Antioxidants attenuate gentamicininduced free radical formation in vitro and ototoxicity in vivo: D-methionine is a potential protectant. Hear Res 142: 34-40, 2000.

65. Kashio A, Sakamoto T, Suzukawa K, Asoh S, Ohta S and Yamasoba T: A protein derived from the fusion of TAT peptide and $\mathrm{FNK}$, a Bcl-x(L) derivative, prevents cochlear hair cell death from aminoglycoside ototoxicity in vivo. J Neurosci Res 85: 1403-1412, 2007.

66. Duan M, Agerman K, Ernfors P and Canlon B: Complementary roles of neurotrophin 3 and a N-methyl-D-aspartate antagonist in the protection of noise and aminoglycoside-induced ototoxicity. Proc Natl Acad Sci USA 97: 7597-7602, 2000.

67. Basile AS, Huang JM, Xie C, Webster D, Berlin C and Skolnick P: N-methyl-D-aspartate antagonists limit aminoglycoside antibioticinduced hearing loss. Nat Med 2: 1338-1343, 1996.

68. Sha SH and Schacht J: Are aminoglycoside antibiotics excitotoxic? Neuroreport 9: 3893-3895, 1998.

69. Oliveira JA, Canedo DM, Rossato M and Andrade MH: Selfprotection against aminoglycoside ototoxicity in guinea pigs. Otolaryngol Head Neck Surg 131: 271-279, 2004.

70. Hester TO, Jones RO and Clerici WJ: Protection against aminoglycoside otic drop-induced ototoxicity by a spin trap: I. Acute effects. Otolaryngol Head Neck Surg 119: 581-587, 1998.

71. Bailey RR, Begg EJ, Smith AH, Robson RA, Lynn KL, Chambers ST, Barclay ML and Hornibrook J: Prospective, randomized, controlled study comparing two dosing regimens of gentamicin/oral ciprofloxacin switch therapy for acute pyelonephritis. Clin Nephrol 46: 183-186, 1996. 